\title{
Glutamate Cysteine Ligase-Modulatory Subunit Knockout Mouse Shows Normal Insulin Sensitivity but Reduced Liver Glycogen Storage
}

\begin{abstract}
Suzie Lavoie ${ }^{1,2 *}$, Pascal Steullet ${ }^{1}$, Anita Kulak ${ }^{1}$, Frederic Preitner ${ }^{3}$, Kim Q. Do ${ }^{1}$ and Pierre J. Magistretti ${ }^{1,4,5}$

${ }^{1}$ Department of Psychiatry, Centre for Psychiatric Neuroscience, Lausanne University Hospital and University of Lausanne, Lausanne-Prilly, Switzerland, ${ }^{2}$ Orygen, The National Centre of Excellence in Youth Mental Health, Centre for Youth Mental Health, The University of Melbourne, Parkville, VIC, Australia, ${ }^{3}$ Mouse Metabolic Evaluation Facility, Center for Integrative Genomics, University of Lausanne, Lausanne, Switzerland, ${ }^{4}$ Laboratory of Neuroenergetics and Cellular Dynamics, Brain Mind Institute, Ecole Polytechnique Fédérale de Lausanne, Lausanne, Switzerland, ${ }^{5}$ BESE Division, King Abdullah University of Sciences and Technology (KAUST), Thuwal, Saudi Arabia
\end{abstract}

OPEN ACCESS

Edited by:

Ovidiu Constantin Baltatu,

Camilo Castelo Branco University,

Brazil

Reviewed by:

Joao Batista Teixeira Rocha, Universidade Federal de Santa Maria,

Brazil

Gregory J. Brewer,

Univeristy of California Irvine, USA

*Correspondence:

Suzie Lavoie

suzie.lavoie@orygen.org.au

Specialty section:

This article was submitted to

Integrative Physiology,

a section of the journal

Frontiers in Physiology

Received: 01 February 2016 Accepted: 04 April 2016

Published: 21 April 2016

Citation:

Lavoie S, Steullet $P$, Kulak $A$ Preitner F, Do KQ and Magistretti PJ

(2016) Glutamate Cysteine

Ligase-Modulatory Subunit Knockout Mouse Shows Normal Insulin Sensitivity but Reduced Liver

Glycogen Storage.

Front. Physiol. 7:142.

doi: 10.3389/fphys.2016.00142
Glutathione (GSH) deficits have been observed in several mental or degenerative illness, and so has the metabolic syndrome. The impact of a decreased glucose metabolism on the GSH system is well-known, but the effect of decreased GSH levels on the energy metabolism is unclear. The aim of the present study was to investigate the sensitivity to insulin in the mouse knockout $(\mathrm{KO})$ for the modulatory subunit of the glutamate cysteine ligase (GCLM), the rate-limiting enzyme of GSH synthesis. Compared to wildtype (WT) mice, GCLM-KO mice presented with reduced basal plasma glucose and insulin levels. During an insulin tolerance test, GCLM-KO mice showed a normal fall in glycemia, indicating normal insulin secretion. However, during the recovery phase, plasma glucose levels remained lower for longer in $\mathrm{KO}$ mice despite normal plasma glucagon levels. This is consistent with a normal counterregulatory hormonal response but impaired mobilization of glucose from endogenous stores. Following a resident-intruder stress, during which stress hormones mobilize glucose from hepatic glycogen stores, $\mathrm{KO}$ mice showed a lower hyperglycemic level despite higher plasma cortisol levels when compared to WT mice. The lower hepatic glycogen levels observed in GCLM-KO mice could explain the impaired glycogen mobilization following induced hypoglycemia. Altogether, our results indicate that reduced liver glycogen availability, as observed in GCLM-KO mice, could be at the origin of their lower basal and challenged glycemia. Further studies will be necessary to understand how a GSH deficit, typically observed in GCLM-KO mice, leads to a deficit in liver glycogen storage.

Keywords: glutathione, GCLM knockout, glycogen, insulin, glycemia, resident-intruder stress, cortisol

Abbreviations: CORT, corticosterone; GCLC, glutamate cysteine ligase catalytic subunit; GCLM, glutamate cysteine ligase modulatory subunit; GSSG, oxidized glutathione; HPA, hypothalamopituitary-adrenal; ITT, Insulin tolerance test; NADPH, nicotinamide adenine dinucleotide phosphate; ROS, reactive oxygen species. 


\section{INTRODUCTION}

Deficits in glutathione, a major regulator of reactive oxygen species (ROS) levels, have been observed in several neurodegenerative disorders including Alzheimer's, Parkinson's, and Huntington's diseases (For reviews see Dringen and Hirrlinger, 2003; Ballatori et al., 2009), as well as in psychiatric illnesses, such as major depressive and bipolar disorders (Gawryluk et al., 2011), and schizophrenia (Yao et al., 2006; Do et al., 2009a,b; Gawryluk et al., 2011). Mental illnesses have repeatedly been associated with the metabolic syndrome (For reviews see Henderson et al., 2015; Vancampfort et al., 2015; Xu et al., 2015), and excess levels of ROS may contribute to the development of those pathologies that have reached epidemic proportions, such as atherosclerosis and Diabetes Mellitus (For a review see Santilli et al., 2015). Therefore, understanding the impact of a chronic glutathione (GSH) deficit on energy metabolism has important clinical implications.

Glucose serves as the principal source of energy in the body. This monosaccharide can be metabolized via two pathways, the glycolysis and the pentose phosphate pathway. Glucose metabolism through the glycolysis pathway is usually followed by the tricarboxylic acid cycle yielding 30 to 36 ATP per glucose (For a review on energy metabolism see Allaman and Magistretti, 2013). Side products of this important energygenerating pathway are reactive oxygen species (For a review see Quijano et al., 2015). ROS are free radical atoms or molecules with an unpaired electron that renders them highly reactive.

Free radicals are essential in several biochemical processes including the regulation of insulin sensitivity and glucose homeostasis (For a review see Bisbal et al., 2010). However, if ROS levels become too high, they can induce significant damage to proteins, membranes and DNA (Halliwell and Chirico, 1993; Halliwell, 1999). The major cellular antioxidant and redox regulator in living cells is the tripeptide glutathione (Orlowski and Karkowsky, 1976; Meister and Anderson, 1983; Dringen, 2000; Hammond et al., 2001). GSH is needed for the reduction of reactive glycolysis by-products. It is also known that low glucose availability is accompanied by severe redox imbalance, partially due to the fact that the metabolism of glucose through the pentose phosphate pathway produces the NADPH necessary to maintain a proper GSH/GSSG redox balance (Pias and Aw, 2002; Tang et al., 2015). Thus, there is a tight link between the GSH system and glucose metabolism.

However, the way a primary GSH deficit can impact on glucose metabolic pathways remains unclear. The effects of oxidative stress and elevated free radicals on glucose metabolism have so far mainly been studied in the context of obesity and diabetes. These studies have demonstrated that high-fat diet and obesity lead to excessive production of ROS, which in turn contribute to insulin resistance (for reviews see Bashan et al., 2009; Bisbal et al., 2010). Moreover, a lower GSH/GSSG ratio and associated oxidative stress have been demonstrated to precede an increase in insulin resistance and impairment in glucose homeostasis (Paolisso et al., 1992; Nwose et al., 2006). In contrast, a study using GSH peroxidase knockout mice, which are characterized by redox imbalance and increased ROS levels, resulted in enhanced insulin sensitivity in these animals (Loh et al., 2009). The contribution of a GSH dysregulation in insulin resistance remains unclear.

A valuable model to study the effect of a chronic GSH deficit and the consequent chronic vulnerability to oxidative stress is the mouse knockout (KO) for the modulatory subunit of the glutamate cysteine ligase (GCLM), the rate-limiting enzyme of GSH synthesis (Yang et al., 2002; Lavoie et al., 2009; Steullet et al., 2010). These mice present with severe and chronic GSH deficit of $80 \%$ and more in liver, lung, kidney, pancreas, and plasma (Yang et al., 2002; McConnachie et al., 2007), as well as in brain (McConnachie et al., 2007; Steullet et al., 2010) and brain cells (Giordano et al., 2006; Lavoie et al., 2009). GCLM-KO mice also show increased oxidative stress markers levels (Kendig et al., 2011). Kendig et al. (2011) have demonstrated that GCLM-KO mice fed a high fat diet, are protected against the development of diet-induced obesity, glucose intolerance, and insulin resistance (as assessed by the HOMA-IR index, i.e., the product of fasted insulinemia X glycemia). Under normal chow feeding, GCLMKO mice showed a normal HOMA-IR index, suggesting normal insulin sensitivity although this was not formally confirmed with tests assessing insulin sensitivity.

The present study therefore aimed to investigate the sensitivity to insulin in the GCLM-KO mouse. We hypothesized that under normal chow feeding, GCLM-KO mice would present with normal insulin sensitivity. Basal plasma glucose and insulin were measured, and insulin tolerance tests were performed. Based on the results obtained, exploratory experiments were conducting during which the counterregulatory hormone glucagon was measured as well and hepatic glycogen stocks. The glucose response to an acute resident-intruder stress was also assessed.

\section{MATERIALS AND METHODS}

\section{Animals}

GCLM-KO mice, back crossed with C57BL/6J mice over more than 10 generations, were kindly provided by Timothy P. Dalton and Ying Chen (Center for Environmental Genetics, Cincinnati, OH, USA; Yang et al., 2002). Male mice used for the present study were bred in the local animal facility under normal 12:12-h light/dark cycle. Mice were gently handled daily for 1 week prior to experiments in order to habituate them to manipulation and minimize the stress induced by handling/manipulation during injections and blood collections. All experiments were performed in accordance with the guidelines outlined in the Guide for the Care and Use of Laboratory Animals (National Research Council) and were approved by the Consumer and Veterinary Affairs Services of the Canton Vaud, Switzerland.

\section{Material}

Unless otherwise stated, all chemicals were purchased from Sigma-Aldrich (St-Louis, MO, USA).

\section{Methods}

Before all experiments, WT and KO mice were single-housed overnight for $16 \mathrm{~h}$ and food-restricted during the last $4 \mathrm{~h}$ in order to reach a stable glycemic state. Samples were collected 
during the animals' light phase between 12:00 and 14:00 h for all experiments.

\section{Basal Plasma Glucose Levels}

Blood samples were obtained from tail-tip bleedings for immediate glycemia measurements with a glucometer (Ascensia Breeze2, Bayer AG, Leverkusen, Germany).

\section{Basal Plasma Insulin Levels}

With the tail nick procedure, blood was collected with Microvette capillary tubes EDTA-2Na (Sarstedt, Nümbrecht, Germany). Blood was then immediately centrifuged $\left(4^{\circ} \mathrm{C}, 10000 \mathrm{rpm}\right.$, $15 \mathrm{~min}$ ) and the plasma was frozen at $-20^{\circ} \mathrm{C}$ until measurements. Insulin levels were quantified with a commercially available Insulin enzyme immunoassay kit (Alpco Immunoassays, Salem, NH, USA).

\section{Insulin Tolerance Test}

Mice were i.p.-injected with insulin $(0.5 \mathrm{U} / \mathrm{kg}$, diluted in BSA 0.5\%; Actrapid, Novo Nordisk Pharma SA, Küsnacht, Switzerland) at around 13:00 (corresponding to 4-h fasting). Blood samples were obtained from tail-tip bleedings at the time of injection (time $=0$ ) and 15, 30, 60, 90, and $120 \mathrm{~min}$ after injection. Plasma glucose levels were measured with a glucometer.

\section{Plasma Glucagon Levels}

Since large blood quantities (at least $100 \mu$ l of plasma) were necessary for glucagon measurements, animals were decapitated and trunk blood was collected with Microvette capillary tubes EDTA-2Na, to which Aprotinin was added, and was immediately centrifuged $\left(4^{\circ} \mathrm{C}, 10000 \mathrm{rpm}, 15 \mathrm{~min}\right)$. Plasma extracted was immediately frozen at $-80^{\circ} \mathrm{C}$ and subsequently unfrozen for glucagon levels measurements with the Glucagon enzyme immunoassay kit (Alpco Immunoassays, Salem, NH, USA).

\section{Hepatic Glycogen Levels}

Mice were decapitated. The liver was rapidly extracted, immediately frozen on carbon dioxide ice and then kept at $-80^{\circ} \mathrm{C}$. For glycogen measurements, frozen samples were placed into Eppendorf tubes and weighed before $\mathrm{NaOH} 0.1 \mathrm{M}$ was added to stop enzyme activity. Samples were homogenized on ice and a $50-\mu l$ aliquot was used to measure the protein content using the BCA protein assay reagent kit (Pierce, Rockford, IL, USA). Tubes were then centrifuged at $14000 \mathrm{~g}$ for $10 \mathrm{~min}$ and the supernatant was used for glycogen dosage following a previously described procedure (Allaman et al., 2010). In a first $100-\mu \mathrm{l}$ aliquot, $300 \mu \mathrm{l}$ of sodium-acetate buffer $(0.1$ $\mathrm{M}, \mathrm{pH} 4.6)$ was added. In the second one, $300 \mu \mathrm{l}$ of the same buffer containing $1 \%(\mathrm{v} / \mathrm{v})$ of amyloglucosidase $(10 \mathrm{mg} / \mathrm{ml}$; Roche Diagnostics, Rothkreuz, Switzerland) was added. Aliquots were incubated at room temperature (RT) for $30 \mathrm{~min}$. Then, $2 \mathrm{ml}$ of Tris- $\mathrm{HCl}$ buffer (0.1M; pH 8.1; $\mathrm{MgCl}_{2} 3.3 \mathrm{mM}$, ATP $0.2 \mathrm{mM}$, NADP $30 \mu \mathrm{M}$, containing $0.7 \mathrm{U} / \mathrm{ml}$ of hexokinase, and $0.35 \mathrm{U} / \mathrm{ml}$ of glucose 6-phosphate dehydrogenase (Roche Diagnostics)) were added, and the mixture was incubated at RT for $30 \mathrm{~min}$. Fluorescence associated with the NADPH formed was then read on a fluorimeter (excitation: $340 \mathrm{~nm}$; emission: $450 \mathrm{~nm}$ ) after calibration with an appropriate standard curve using glucose as standard. The first aliquot gives the sum of glucose and glucose 6-phosphate, and the second gives the sum of glycogen, glucose, and glucose-6-phosphate; the amount of glycogen was determined by subtracting the result obtained from the first aliquot from the result obtained from the second aliquot. Results are presented in nmol glycogen per mg of protein, one mole of glycogen corresponding to one mole of glycosyl units originating from glycogen.

\section{Resident-Intruder Stress}

An adapted version of the resident-intruder paradigm (Martinez et al., 1998; Heinrichs and Koob, 2005) was used to induce stress 1 month after the ITT. For the stress procedure, a weight-matched white OF1 (Charles River, L'Arbresle, France) intruder mouse was placed into the cage of the black WT or GCLM-KO resident for a period of $30 \mathrm{~min}$. Plasma glucose levels were measured immediately before and after the stress.

Plasma corticosterone (CORT) levels were measured before the resident-intruder stress, immediately after the stress and $60 \mathrm{~min}$ after to assess the hormonal response immediately after the stress and during the remission period. Between 20 and $30 \mu \mathrm{l}$ of blood was sampled using the tail-nick procedure and collected with a lithium-heparin coated capillary tube (Microvette CB 300, Sarstedt, Nuembrecht, Germany). Blood samples were centrifuged ( $4^{\circ} \mathrm{C}, 4000 \mathrm{rpm}, 15 \mathrm{~min}$ ) before plasma was extracted and stored at $-20^{\circ} \mathrm{C}$ until further processing. Blood was unfrozen for CORT levels measurements using the Corticosterone enzyme immunoassay kit (Assay Designs, Ann Arbor, MI, USA).

\section{Statistical Analyses}

Statistical analyses were performed using SPSS Statistics 17.0 (Chicago, IL, USA). For comparisons between WT and KO, the $t$-test for independent samples was used. Significant probability level was set to $p \leq 0.05$. For the ITT, because multiple measurements were taken overtime, repeated-measure ANOVAs with Time as within-subject factor and Genotype as between factor were performed. The effect of time was then assessed with paired-samples $t$-test between each time point and time $=0$, and the Bonferroni correction for multiple comparisons was used. The effect of genotype was assessed with $t$-tests for independent samples at each time point, also Bonferronicorrected for multiple comparisons. CORT levels measured $60 \mathrm{~min}$ after the end of stress were not distributed normally, so the non-parametric Mann-Whitney test for independent samples was used to compare between genotypes at this time point and the Wilcoxon signed-rank test was used to assess the difference within each genotype over time.

\section{RESULTS}

\section{Lower Basal Plasma Glucose and Insulin Levels in GCLM-KO Mice}

To establish the basal experimental condition, plasma glucose and insulin levels were measured in both GCLM-KO and -WT mice. 
Following 4-h food deprivation, both plasma glucose and insulin levels were lower in GCLM-KO mice compared to WT $(-9.9 \%, p=0.015 ;-56.4 \%, p=0.01$, respectively; Figure 1). We reasoned that this might be due to either a primary hypoglycemia with a secondary hypoinsulinemia, and/or a primary action of insulin to more efficiently lower glycemia. In order to assess this last hypothesis, and to verify our primary hypothesis that insulin tolerance is normal in GLCM-KO mice, we assessed insulin action by an insulin tolerance test.

\section{Normal Insulin Sensitivity but Delayed Recovery from Insulin-Induced Hypoglycemia in GCLM-KO Mice}

At the time of the insulin tolerance test (ITT) 3 months-old GLCM-KO males were lighter than WT $(23.8 \pm 0.4$ and $26.8 \pm$ 0.4 g respectively; $-13 \% ; p<0.001$ ).

Figure 2 shows that, as seen previously in Figure 1, basal glycemia (time $=0$ ) tended to be lower in GCLM-KO mice (raw $p$-value $=0.023$, although it did not reach statistical significance when Bonferroni-corrected for multiple measures in this test). Repeated-measure ANOVA showed a significant within-subject effect of time $\left[F_{(5,17)}=93.229 ; p<0.001\right]$, and a significant interaction between Time and Genotype $\left[F_{(1)}=3.559\right.$; $p=0.008$ ]. Specifically, The insulin bolus decreased plasma glucose to a similar extent in both WT and GCLM-KO mice up to $30 \mathrm{~min}$ post-bolus, suggesting that GCLM-KO mice have normal insulin sensitivity. However, during the phase of recovery from hypoglycemia, plasma glucose levels remained significantly lower in GCLM-KO mice compared to WT at times $60 \min (-27.9 \% ; p=0.001), 90 \min (-44.6 \% ; p=0.001)$, and $120 \mathrm{~min}(-41.2 \% ; p=0.004)$ post-insulin-injection. These results are suggestive of an impaired counterregulation in KO mice. To investigate this hypothesis, levels of glucagon, the major participant in the counterregulation, were measured.

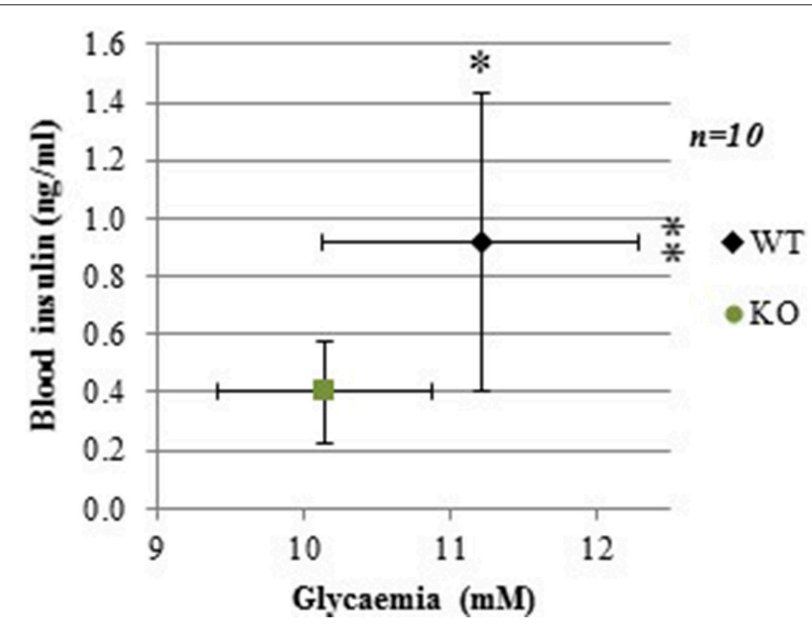

FIGURE 1 | Lower basal plasma glucose and insulin levels in GCLM-KO mice compared to WT mice. Data are expressed as mean \pm SD. ${ }^{*} p<0.05$; ${ }^{* *} p<0.01$.

\section{Normal Plasma Levels of the Counterregulatory Hormone Glucagon}

Sixty minutes after insulin injection, at the time when glucose levels started to increase back to basal levels in WT mice (Figure 2), plasma glucagon levels were not significantly different between WT and KO mice (Figure 3). In absence of counterregulatory defect at the hormonal level, the delayed recovery from insulin-induced hypoglycemia could be due to an inability to mobilize glucose from endogenous stores. To assess this hypothesis, another glucose-mobilizing paradigm was tested, i.e., acute stress-induced hyperglycemia where stress hormones such as corticosterone mediate the mobilization of glucose from hepatic glycogen.

\section{Lower Glycemic Levels in Response to Acute Social Stress Despite Higher CORT Response}

The acute social stress paradigm was used as another experimental challenge known to increase glycemia through
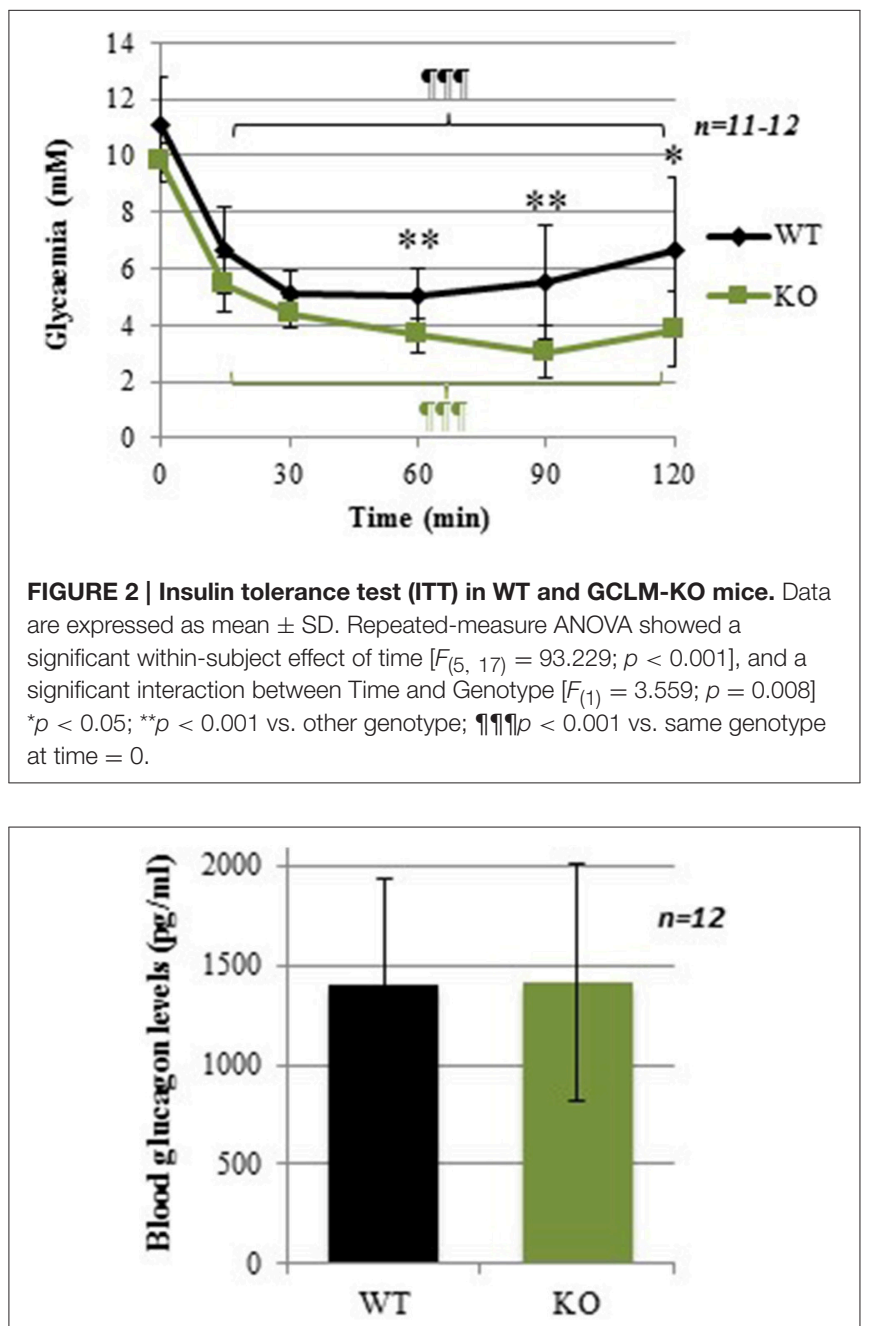

FIGURE 3 | Plasma glucagon levels in WT and GCLM-KO mice $60 \mathrm{~min}$ after insulin injection. Data are expressed as mean \pm SD. 
mobilization of hepatic glycogen. Before stress induction, plasma glucose levels were lower in $\mathrm{KO}$ compared to WT (Figure 4A; $-15.5 \% ; p=0.043$ ). After stress, plasma glucose levels were increased in both WT $(1.26$-fold increase; $p<0.001)$ and KO mice (1.25-fold increase; $p<0.001)$, but the difference between the two genotypes remained the same (Figure 4A; $-13.3 \%$; $p=0.034)$. These results show that during an acute social stress, GCLM-KO mice are unable to increase glycemia at the same levels as WT mice.

Levels of the stress hormone corticosterone (CORT), which elevation mediates the stress-induced increase in glycemia, were measured before stress, immediately after stress, as well as $60 \mathrm{~min}$ after stress induction. At baseline, CORT levels were similar in WT and KO mice (Figure 4B). These levels were higher immediately after the stress period in both WT (8.4-fold increase; $p<0.001)$ and KO (7.5-fold increase; $p<0.001)$, with higher values in $\mathrm{KO}$ when compared to WT mice $(+24 \% ; p=0.043)$. Sixty minutes after the end of the stress, CORT levels showed no more difference with baseline in WT, while they were still

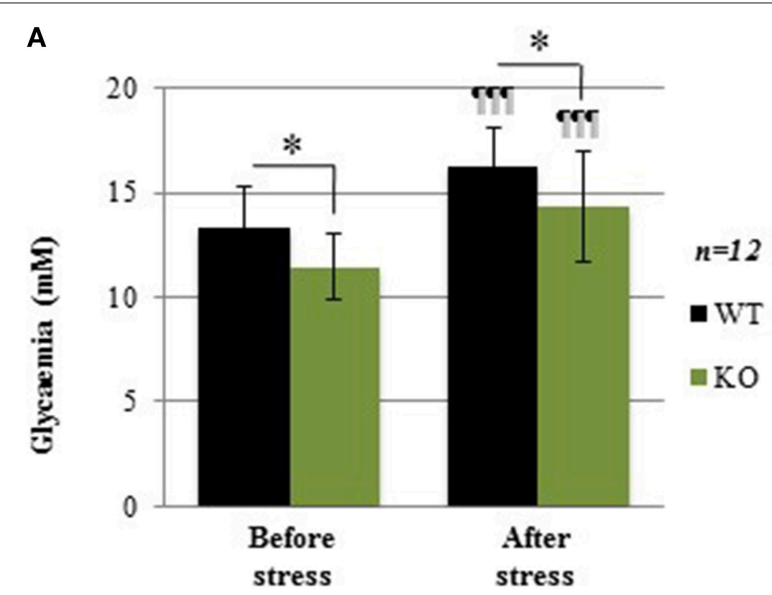

B

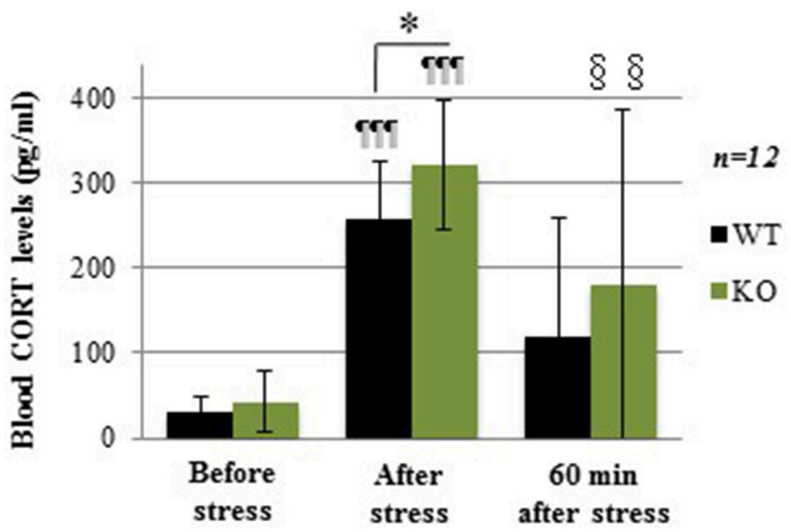

FIGURE 4 | (A) Glycemia in WT and GCLM-KO mice before and after a 30-min resident-intruder stress. (B) Plasma corticosterone levels in WT and KO mice before, immediately after the stress and 60 min later. Data are expressed as mean \pm SD. CORT levels were higher just after the stress period in both WT (8.4-fold increase; $p<0.001)$ and KO (7.5-fold increase; $p<0.001)$. ${ }^{*} p<0.05$ vs. other genotype; १ी१ $p<0.001$ vs. same genotype immediately before stress induction (baseline). $\S \S p<0.01$ vs. same genotype at baseline. higher in $\mathrm{KO}(p=0.004)$.In summary, during acute stress, although the stress-induced hormonal response was stronger in GCLM-KO mice compared to WT mice, glycemia in $\mathrm{KO}$ mice did not rise as high as in WT. The negative feedback control of CORT was not as robust in $\mathrm{KO}$ mice. Thus, overall, this result is more consistent with the hypothesis of alterations in glycogen stores rather than with an impaired ability of effector pathways to mobilize glucose from glycogen. To test this hypothesis, glycogen levels were measured.

\section{Lower Hepatic Glycogen Content in GCLM-KO Mice}

Hepatic glycogen content was measured in mice after a $4 \mathrm{~h}$ fasting period. Consistent with our hypothesis, GCLM-KO mice showed a strikingly lower glycogen hepatic content compared to WT (-48.7\%; $p=0.008$; Figure 5).

\section{DISCUSSION}

The present study shows that GCLM-KO mice present with lower plasma glucose and insulin levels, and a reduced ability to increase plasma glucose in response to insulin-induced hypoglycemia or to acute stress. These metabolic alterations are associated with lower levels of hepatic glycogen when compared to WT mice.

During the first $30 \mathrm{~min}$ of the ITT, the decrease in glucose in response to insulin was comparable between the two genotypes suggesting normal insulin sensitivity. After $60 \mathrm{~min}$, when glycemia started to increase back to baseline level in WT mice, it remained lower in $\mathrm{KO}$ mice. As the half-life of insulin is about $10 \mathrm{~min}$ in mice (Cresto et al., 1977), late differences in glucose concentration between groups (beyond $30 \mathrm{~min}$ after the insulin bolus) are not likely to reflect alterations in insulin action (Ayala et al., 2010). Thus, ITT results do not speak in favor of abnormal insulin sensitivity, as opposed to the impaired insulin sensitivity previously observed in other models of impaired GSH homeostasis (Paolisso et al., 1992; Nwose et al., 2006; Loh et al., 2009). It can be hypothesized that animals presenting chronic low antioxidant capacity have developed compensatory

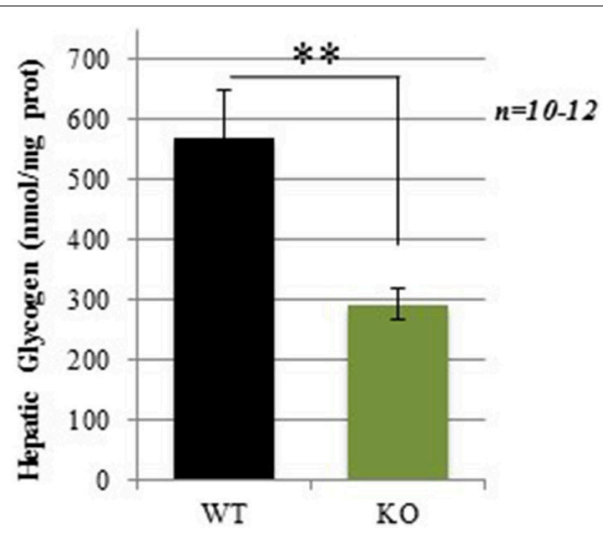

FIGURE 5 | Hepatic glycogen levels in WT and GCLM-KO mice. Data are expressed as mean $\pm \mathrm{SD}$. ${ }^{* *} p<0.001$ 
mechanisms to prevent such a metabolic dysregulation. Our ITT results also suggest that GCLM-KO animals display impaired counterregulatory mobilization of glucose from endogenous stores.

Compromised counterregulatory response to hypoglycemia could be due to the decrease in counterregulatory hormones released. In response to low plasma glucose levels, catecholamines, pancreatic glucagon, growth hormones, and cortisol are released, resulting in a stimulation of hepatic glycogenolysis (For a review see Amiel, 1991). Plasma levels of glucagon, the most important hormone involved in achieving recovery of glucose levels following acute hypoglycemia (Rizza et al., 1979), were similar in WT and KO mice $60 \mathrm{~min}$ after insulin injection (time when WT mice had started to normalize their glycemia). This indicates that the hormonal counterregulatory response to hypoglycemia is normal in $\mathrm{KO}$ mice. It cannot be excluded that glucagon signaling, or the activity of other hormones involved in the counterregulation may be compromised in these mice.

Compromised counterregulation might stem from an alteration in glycogen stores. Supporting this hypothesis is the observed lower hepatic glycogen levels found in $\mathrm{KO}$ compared to WT mice. Interestingly, in cultures of astrocytes from the GCLM-KO mice, lower basal glycogen levels and a decrease in its mobilization after an oxidative stress were observed when compared to astrocytes from WT mice (Lavoie et al., 2011). In vivo, already after 2 or $3 \mathrm{~h}$ of food removal, hepatic glycogen is usually reduced in mice (Baker and Huebotter, 1972; Seyer et al., 2013), meaning that hepatic glycogen is required for maintenance of euglycemia even shortly after food removal. Thus, lower glycogen availability and/or mobilization could be responsible for the impaired counterregulatory response during experimental hypoglycemia in KO mice. How the lack of GCLM leads to a deficiency of hepatic glycogen remains unclear.

Consistent with the role of altered hepatic glycogen stores in the counterregulatory response, the hepatic glycogen-dependent hyperglycemic response to social stress was also altered in GCLM-KO mice. When a physical or psychological stress occurs, the hypothalamic-pituitary-adrenal (HPA) axis is activated, leading to an increase in circulating glucocorticoids (For a review see Chrousos and Gold, 1992) that are known to modulate glucose metabolism. Our results clearly show that after a stress induced by the presentation of an intruder mouse for $30 \mathrm{~min}$, CORT levels increased considerably in both WT and KO mice. Interestingly, the stress-induced hormonal response was stronger in GCLM-KO mice compared to WT mice indicating that the negative feedback control of CORT was less efficient in $\mathrm{KO}$ mice. However, the higher CORT levels in stressed KO mice did not lead to higher glycemia in these mice as compared to stressed WT mice. Therefore, even though the CORT response was supranormal, the stress-induced hyperglycemia itself appears to be compromised. This observation is in line with the hypothesis of the importance of reduced glycogen availability in the alteration of glucose homeostasis in $\mathrm{KO}$ mice although further studies to establish causality are warranted.

Finally, it is worth noting that this study reveals another physiological dysregulation/adaptation in GCLM-KO mice, namely an attenuated negative feedback regulation of CORT, which was also observed following a synthetic cortisol (dexamethasone) injection (unpublished observation). This observation is similar to the attenuated hormonal negative feedback response reported in patients with schizophrenia or bipolar disorder when pharmacologically challenged with dexamethasone (Mück-Šeler et al., 1999; Watson et al., 2004). It has been shown that oxidative stress induced by hyperoxia in rats led to a loss of glucocorticoid receptors in the hippocampus resulting in an elevation of the HPA activity due a decrease in the feedback regulation of the HPA axis (Kobayashi et al., 2009).

GCLM-KO mice present with chronically low GSH levels and increased oxidative stress markers levels. Knowing that the end result of glucose metabolism is accompanied by the production of reactive oxygen species, it has been postulated that adaptation toward oxidative stress in GCLM-KO mice may partly involve a constraint/limitation of glucose utilization and glycogen mobilization when an oxidative challenge is already monopolizing the GSH system (Lavoie et al., 2011). On the other hand, GCLM-KO mice present with lower weight, plasma glucose and insulin and hepatic glycogen levels compared to WT, observations consistent, among other possibilities, with a faster metabolism (Kendig et al., 2011). In this case, more ROS would be produced which would put more burden on the already deficient antioxidant system of the GCLM-KO, unless these mice switched from glycolysis (production of ROS) to the pentose phosphate pathway (generation of NADPH) as suggested by Ralser et al. (2007). In the light of the current literature, it remains unclear which energy pathway is favored by mice showing chronic oxidative stress, but it has been suggested that NADPH generation may be a more efficacious therapeutic target upstream of GSH and ROS (Ghosh et al., 2014).

\section{CONCLUSION}

Our results indicate that GCLM-KO mice do not show impaired sensitivity to insulin contrary to other GSH-deficient mouse models. The GCLM-KO mice presented with reduced liver glycogen availability that could be at the origin of their lower basal and challenged glycemia, even in the present of normal levels of hyperglycemiant hormones (i.e., glucagon following insulin-induced hypoglycaemia and corticosterone following acute social stress). Further studies are warranted to assess the direct association between the deficit in GSH associated with GCLM targeted deletion and glycogen storage not only in the liver but also in other organs in both fed and fasted conditions.

\section{AUTHOR CONTRIBUTIONS}

SL has contributed to the design of the work, as well as the acquisition, analysis, and interpretation of data for the work. She has drafted the work and approved the final version to be published. PS has contributed to the design of the work and to the interpretation of data for the work. He has critically revised the manuscript and approved the final version to be published. $\mathrm{AK}$ and FP have contributed to the acquisition and interpretation of data for the work. They have critically revised the manuscript and approved the final version to be published. KD and PM have 
contributed to the design of the work, and they have critically revised the manuscript and approved the final version to be published. All authors agree to be accountable for all aspects of the work in ensuring that questions related to the accuracy or integrity of any part of the work are appropriately investigated and resolved.

\section{FUNDING}

This work was financially supported by the Swiss National Foundation No 310000-116689 and the MTR schizophrenia

\section{REFERENCES}

Allaman, I., Gavillet, M., Bélanger, M., Laroche, T., Viertl, D., Lashuel, H. A., et al. (2010). Amyloid-beta aggregates cause alterations of astrocytic metabolic phenotype: impact on neuronal viability. J. Neurosci. 30, 3326-3338. doi: 10.1523/JNEUROSCI.5098-09.2010

Allaman, I., and Magistretti, P. J. (2013). "Brain energy metabolism," in Fundamentals in Neuroscience, 4th Edn., eds B. Squire, L. D. Bloom, F. E. Du Lac, S. Ghosh, A. Spitzer (San Diego, CA: Academic Press), 261-284. doi: 10.1016/B978-0-12-385870-2.00012-3

Amiel, S. (1991). Glucose counter-regulation in health and disease: current concepts in hypoglycaemia recognition and response. Q. J. Med. 80, 707-727.

Ayala, J. E., Samuel, V. T., Morton, G. J., Obici, S., Croniger, C. M., Shulman, G. I., et al. (2010). Standard operating procedures for describing and performing metabolic tests of glucose homeostasis in mice. Dis. Model. Mech. 3, 525-534. doi: $10.1242 / \mathrm{dmm} .006239$

Baker, N., and Huebotter, R. J. (1972). Rapid activation and inactivation of fatty acid synthesis from glucose in vivo. J. Lipid Res. 13, 329-337.

Ballatori, N., Krance, S. M., Notenboom, S., Shi, S., Tieu, K., and Hammond, C. L. (2009). Glutathione dysregulation and the etiology and progression of human diseases. Biol. Chem. 390, 191-214. doi: 10.1515/BC.2009.033

Bashan, N., Kovsan, J., Kachko, I., Ovadia, H., and Rudich, A. (2009). Positive and negative regulation of insulin signaling by reactive oxygen and nitrogen species. Physiol. Rev. 89, 27-71. doi: 10.1152/physrev.00014.2008

Bisbal, C., Lambert, K., and Avignon, A. (2010). Antioxidants and glucose metabolism disorders. Curr. Opin. Clin. Nutr. Metab. Care 13, 439-446. doi: 10.1097/MCO.0b013e32833a5559

Chrousos, G. P., and Gold, P. W. (1992). The concepts of stress and stress system disorders. Overview of physical and behavioral homeostasis. JAMA 267, 1244-1252. doi: 10.1001/jama.1992.03480090092034

Cresto, J. C., Lavine, R. L., Buchly, M. L., Penhos, J. C., Bhathena, S. J., and Recant, L. (1977). Half life of injected 125I-insulin in control and ob/ob mice. Acta Physiol. Lat. Am. 27, 7-15.

Do, K. Q., Bovet, P., Cabungcal, J. H., Conus, P., Gysin, R., Lavoie, S., et al. (2009a). "Redox dysregulation in schizophrenia: Genetic susceptibility and pathophysiological mechanisms," in Hankbook of Neurochemistry and Molecular Neurobiology, 3rd Edn., eds D. C. Javitt and J. T. Kantrowitz (New York, NY: Springer), 285-311. doi: 10.1007/978-0-387-30410-6_8

Do, K. Q., Cabungcal, J. H., Frank, A., Steullet, P., and Cuenod, M. (2009b). Redox dysregulation, neurodevelopment, and schizophrenia. Curr. Opin. Neurobiol. 19, 220-230. doi: 10.1016/j.conb.2009.05.001

Dringen, R. (2000). Metabolism and functions of glutathione in brain. Prog. Neurobiol. 62, 649-671. doi: 10.1016/S0301-0082(99)00060-X

Dringen, R., and Hirrlinger, J. (2003). Glutathione pathways in the brain. Biol. Chem. 384, 505-516. doi: 10.1515/BC.2003.059

Gawryluk, J. W., Wang, J. F., Andreazza, A. C., Shao, L., and Young, L. T. (2011). Decreased levels of glutathione, the major brain antioxidant, in postmortem prefrontal cortex from patients with psychiatric disorders. Int. J. Neuropsychopharmacol. 14, 123-130. doi: 10.1017/S1461145710000805

Ghosh, D., Levault, K. R., and Brewer, G. J. (2014). Relative importance of redox buffers GSH and $\mathrm{NAD}(\mathrm{P}) \mathrm{H}$ in age-related neurodegeneration and Alzheimer of the Department of Psychiatry of the Lausanne University Hospital and the EPFL.

\section{ACKNOWLEDGMENTS}

The authors would like to thank Anabela Pimentel from the Mouse Metabolic Evaluation Facility of the University of Lausanne for her implication in the ITTs. We would also like to acknowledge Dr Igor Allaman and Dr Jean-Marie Petit for their critical comments on the manuscript. The authors are also grateful to Adeline Cottier and Joel Gyger for their technical assistance.

disease-like mouse neurons. Aging Cell 13, 631-640. doi: 10.1111/acel. 12216

Giordano, G., White, C. C., McConnachie, L. A., Fernandez, C., Kavanagh, T. J., and Costa, L. G. (2006). Neurotoxicity of domoic Acid in cerebellar granule neurons in a genetic model of glutathione deficiency. Mol. Pharmacol. 70, 2116-2126. doi: 10.1124/mol.106.027748

Halliwell, B. (1999). Oxygen and nitrogen are pro-carcinogens. Damage to DNA by reactive oxygen, chlorine and nitrogen species: measurement, mechanism and the effects of nutrition. Mutat. Res. 443, 37-52. doi: 10.1016/S13835742(99)00009-5

Halliwell, B., and Chirico, C. (1993). Lipid peroxidation: its mechanism, measurement, and significance. Am. J. Clin. Nutr. 57, 715S-724S.

Hammond, C. L., Lee, T. K., and Ballatori, N. (2001). Novel roles for glutathione in gene expression, cell death, and membrane transport of organic solutes. J. Hepatol. 34, 946-954. doi: 10.1016/S0168-8278(01)00037-X

Heinrichs, S. C., and Koob, G. F. (2005). Application of experimental stressors in laboratory rodents. Curr. Protoc. Neurosci. 34, 8.4.1-8.4.17. doi: 10.1002/0471142301.ns0804s34

Henderson, D. C., Vincenzi, B., Andrea, N. V., Ulloa, M., and Copeland, P. M. (2015). Pathophysiological mechanisms of increased cardiometabolic risk in people with schizophrenia and other severe mental illnesses. Lancet Psychiatry 2, 452-464. doi: 10.1016/S2215-0366(15)00115-7

Kendig, E. L., Chen, Y., Krishan, M., Johansson, E., Schneider, S. N., Genter, M. B., et al. (2011). Lipid metabolism and body composition in Gclm (-/-) mice. Toxicol. Appl. Pharmacol. 257, 338-348. doi: 10.1016/j.taap.2011.09.017

Kobayashi, N., Machida, T., Takahashi, T., Takatsu, H., Shinkai, T., Abe, K., et al. (2009). Elevation by oxidative stress and aging of hypothalamic-pituitaryadrenal activity in rats and its prevention by vitamin e. J. Clin. Biochem. Nutr. 45, 207-213. doi: 10.3164/jcbn.09-33

Lavoie, S., Allaman, I., Petit, J. M., Do, K. Q., and Magistretti, P. J. (2011). Altered glycogen metabolism in cultured astrocytes from mice with chronic glutathione deficit; relevance for neuroenergetics in schizophrenia. PLoS ONE 6:e22875. doi: 10.1371/journal.pone.0022875

Lavoie, S., Chen, Y., Dalton, T. P., Gysin, R., Cuénod, M., Steullet, P., et al. (2009). Curcumin, quercetin, and tBHQ modulate glutathione levels in astrocytes and neurons: importance of the glutamate cysteine ligase modifier subunit. J. Neurochem. 108, 1410-1422. doi: 10.1111/j.1471-4159.2009.05908.x

Loh, K., Deng, H., Fukushima, A., Cai, X., Boivin, B., Galic, S., et al. (2009). Reactive oxygen species enhance insulin sensitivity. Cell Metab. 10, 260-272. doi: 10.1016/j.cmet.2009.08.009

Martinez, M., Calvo-Torrent, A., and Pico-Alfonso, M. A. (1998). Social defeat and subordination as models of social stress in laboratory rodents: a review. Aggress. Behav. 24, 241-256.

McConnachie, L. A., Mohar, I., Hudson, F. N., Ware, C. B., Ladiges, W. C., Fernandez, C., et al. (2007). Glutamate cysteine ligase modifier subunit deficiency and gender as determinants of acetaminophen-induced hepatotoxicity in mice. Toxicol. Sci. 99, 628-636. doi: 10.1093/toxsci/kfm 165

Meister, A., and Anderson, M. E. (1983). Glutathione. Annu. Rev. Biochem. 52, 711-760. doi: 10.1146/annurev.bi.52.070183.003431

Mück-Šeler, D., Pivac, N., Jakovljević, M., and Brzović, Z. (1999). Platelet serotonin, plasma cortisol, and dexamethasone suppression 
test in schizophrenic patients. Biol. Psychiatry 45, 1433-1439. doi: 10.1016/S0006-3223(98)00174-7

Nwose, E. U., Jelinek, H. F., Richards, R. S., and Kerr, P. G. (2006). Changes in the erythrocyte glutathione concentration in the course of diabetes mellitus. Redox Rep. 11, 99-104. doi: 10.1179/135100006X116583

Orlowski, M., and Karkowsky, A. (1976). Glutathione metabolism and some possible functions of glutathione in the nervous system. Int. Rev. Neurobiol. 19, 75-121. doi: 10.1016/S0074-7742(08)60702-3

Paolisso, G., Di Maro, G., Pizza, G., D’amore, A., Sgambato, S., Tesauro, P., et al. (1992). Plasma GSH/GSSG affects glucose homeostasis in healthy subjects and non-insulin-dependent diabetics. Am. J. Physiol. 263, E435-440.

Pias, E. K., and Aw, T. Y. (2002). Apoptosis in mitotic competent undifferentiated cells is induced by cellular redox imbalance independent of reactive oxygen species production. FASEB J. 16, 781-790. doi: 10.1096/fj. 01-0784com

Quijano, C., Trujillo, M., Castro, L., and Trostchansky, A. (2015). Interplay between oxidant species and energy metabolism. Redox Biol. 8, 28-42. doi: 10.1016/j.redox.2015.11.010

Ralser, M., Wamelink, M. M., Kowald, A., Gerisch, B., Heeren, G., Struys, E. A., et al. (2007). Dynamic rerouting of the carbohydrate flux is key to counteracting oxidative stress. J. Biol. 6, 10. doi: 10.1186/jbiol61

Rizza, R. A., Cryer, P. E., and Gerich, J. E. (1979). Role of glucagon, catecholamines, and growth hormone in human glucose counterregulation. Effects of somatostatin and combined alpha- and beta-adrenergic blockade on plasma glucose recovery and glucose flux rates after insulin-induced hypoglycemia. J. Clin. Invest. 64, 62-71. doi: 10.1172/JCI109464

Santilli, F., D'ardes, D., and Davi, G. (2015). Oxidative stress in chronic vascular disease: from prediction to prevention. Vascul. Pharmacol. 74, 23-37. doi: 10.1016/j.vph.2015.09.003

Seyer, P., Vallois, D., Poitry-Yamate, C., Schütz, F., Metref, S., Tarussio, D., et al. (2013). Hepatic glucose sensing is required to preserve beta cell glucose competence. J. Clin. Invest. 123, 1662-1676. doi: 10.1172/JCI65538

Steullet, P., Cabungcal, J. H., Kulak, A., Kraftsik, R., Chen, Y., Dalton, T. P., et al. (2010). Redox dysregulation affects the ventral but not dorsal hippocampus: impairment of parvalbumin neurons, gamma oscillations, and related behaviors. J. Neurosci. 30, 2547-2558. doi: 10.1523/JNEUROSCI.385709.2010

Tang, H. Y., Ho, H. Y., Wu, P. R., Chen, S. H., Kuypers, F. A., Cheng, M. L., et al. (2015). Inability to maintain GSH pool in G6PD-deficient red cells causes futile AMPK activation and irreversible metabolic disturbance. Antioxid. Redox Signal. 22, 744-759. doi: 10.1089/ars.2014.6142

Vancampfort, D., Stubbs, B., Mitchell, A. J., De Hert, M., Wampers, M., Ward, P. B., et al. (2015). Risk of metabolic syndrome and its components in people with schizophrenia and related psychotic disorders, bipolar disorder and major depressive disorder: a systematic review and meta-analysis. World Psychiatry 14, 339-347. doi: 10.1002/wps.20252

Watson, S., Gallagher, P., Ritchie, J. C., Ferrier, I. N., and Young, A. H. (2004). Hypothalamic-pituitary-adrenal axis function in patients with bipolar disorder. Br. J. Psychiatry 184, 496-502. doi: 10.1192/bjp.184.6.496

Xu, W., Tan, L., Wang, H. F., Jiang, T., Tan, M. S., Tan, L., et al. (2015). Metaanalysis of modifiable risk factors for Alzheimer's disease. J. Neurol. Neurosurg. Psychiatr. 86, 1299-1306. doi: 10.1136/jnnp-2015-310548

Yang, Y., Dieter, M. Z., Chen, Y., Shertzer, H. G., Nebert, D. W., and Dalton, T. P. (2002). Initial characterization of the glutamate-cysteine ligase modifier subunit Gclm (-/-) knockout mouse. J. Biol. Chem. 277, 49446-49452. doi: 10.1074/jbc.M209372200

Yao, J. K., Leonard, S., and Reddy, R. (2006). Altered glutathione redox state in schizophrenia. Dis. Markers 22, 83-93. doi: 10.1155/2006/248387

Conflict of Interest Statement: The authors declare that the research was conducted in the absence of any commercial or financial relationships that could be construed as a potential conflict of interest.

Copyright (C) 2016 Lavoie, Steullet, Kulak, Preitner, Do and Magistretti. This is an open-access article distributed under the terms of the Creative Commons Attribution License (CC BY). The use, distribution or reproduction in other forums is permitted, provided the original author(s) or licensor are credited and that the original publication in this journal is cited, in accordance with accepted academic practice. No use, distribution or reproduction is permitted which does not comply with these terms. 